\title{
SYNCHRONIZATION OF PERTURBED NON-LINEAR
}

\section{HAMILTONIANS}

\author{
BRUCE R. MILLER* \\ National Institute of Standards and Technology, Gaithersburg, MD 20899, U.S.A. \\ and \\ VINCENT T. COPPOLA** \\ Department of Aerospace Engineering, \\ University of Michigan, Ann Arbor MI 48109-2109, U.S.A.
}

(Received 6 May, 1992; accepted 13 August, 1992)

\begin{abstract}
We propose a new method based on Lie transformations for simplifying perturbed Hamiltonians in one degree of freedom. The method is most useful when the unperturbed part has solutions in non-elementary functions. A non-canonical Lie transformation is used to eliminate terms from the perturbation that are not of the same form as those in the main part. The system is thus transformed into a modified version of the principal part. In conjunction with a time transformation, the procedure synchronizes the motions of the perturbed system onto those of the unperturbed part.

A specific algorithm is given for systems whose principal part consists of a kinetic energy plus an arbitrary potential which is polynomial in the coordinate; the perturbation applied to the principal part is a polynomial in the coordinate and possibly the momentum.

We demonstrate the strategy by applying it in detail to a perturbed Duffing system. Our procedure allows us to avoid treating the system as a perturbed harmonic oscillator. In contrast to a canonical simplification, our method involves only polynomial manipulations in two variables. Only after the change of time do we start manipulating elliptic functions in an exhaustive discussion of the flows.
\end{abstract}

Key words: Duffing equation, Hamiltonian systems, Lie transformation, non-canonical transformations, perturbation theory, synchronization.

\section{Introduction}

A Hamiltonian system such as

$$
\mathcal{H}=\frac{1}{2} X^{2}+\frac{\alpha}{2} x^{2}+\frac{\beta}{4} x^{4}+\frac{\gamma}{6} x^{6}
$$

where $|\gamma| \ll|\alpha|,|\beta|$, is often treated as a perturbed harmonic oscillator. Indeed, this approach is a quite reasonable one if, in addition, $|\beta| \ll|\alpha|$. But if $|\alpha| \sim|\beta|$, the treatment will be restricted to a small neighborhood of the origin far away from possible equilibria near $x= \pm \sqrt{-\alpha / \beta}$. Furthermore, the treatment must be carried to high order to properly account for the effects of $\beta$.

In fact, when $|\alpha| \sim|\beta|$ the system should be treated as a perturbed Duffing system. And yet, many perturbation methods, among them classical normalizations

* Email: miller@cam.nist.gov (Internet)

** Email: coppola@krylov.engin.umich.edu (Internet) 
(Deprit, 1969), will encounter great difficulties in doing this. To see why this is so, we reexamine the process involved in normalization.

A normalization eliminates all terms from the perturbation which are in the image of the Lie derivative associated with the principal part. The critical step is the partitioning of terms from the perturbation into kernel and image. The generator of the transformation is then obtained by computing the inverse of the Lie derivative, a step typically involving integration.

The partitioning is clarified by adopting variables which reflect the solutions of the unperturbed part. One then works in the algebra of the solutions, rather than that of the Hamiltonian. This is not a severe problem when only trigonometric solutions are involved. However, non-elementary functions - such as the elliptic functions which solve the Duffing system - present great difficulties, indeed.

The general manipulations of elliptic functions are awkward enough but the partitioning of terms is worse. The final straw, however, is that they are not closed over integration. Since the results of each order of a normalization are folded into the next order, this lack of closure stops the process at some finite order of approximation. The same problem occurs with averaging non-Hamiltonian systems (Coppola, 1989), where the treatment is limited to second order.

An indication of an alternative can be found in a recent sequence of articles in Celestial Mechanics. Howland (1988a; 1988b), and Henrard and Wauthier (1988) considered the subject of a perturbed pendulum resulting from the Ideal Resonance Problem. Eventually, Howland showed how the system could be simplified by eliminating all terms from the perturbation except those found in the pendulum itself. This could be done without explicit manipulations of the elliptic functions. However, a non-canonical transformation was required to achieve such a strong simplification. Henrard and Wauthier followed by showing how canonical differential equations could be recovered by a time transformation derived from the generator of the simplification. We would like to abstract from their work the properties and strengths of a general simplification strategy.

We propose a strategy whereby a normalization is replaced by a two-step process. The first step is a Lie simplification for the purpose of eliminating all terms from the perturbation which are not of the same form as those already in the principal part. Since the computation is carried out in the algebra of the Hamiltonian itself, rather than the algebra of the solutions, it will show its strengths for systems whose principal part has solutions in terms of non-elementary functions, such as (1). After such a simplification, what remains of the perturbation can be collapsed into the main part by altering the coefficients; the simplification replaces the perturbed system with a modified version of the principal part. The second step establishes the time transformation. Although the solutions do become involved at this stage, the computations require only a single integration, avoiding the problems a normalization has with repeated integrations. In effect, this combination of transformations acts as a synchronization of the motions of the perturbed system onto the motions of the unperturbed part via a non-linear clock. 
As another specific example of this general strategy, we offer an algorithm to simplify a broad class of polynomial Hamiltonians in one degree of freedom. In particular, we consider systems whose main part is a kinetic energy plus a potential:

$$
\mathcal{H}_{0}(x, X ; \alpha)=\frac{1}{2} X^{2}+\mathcal{V}(x ; \alpha), \quad \mathcal{V}(x ; \alpha)=\sum_{i=l}^{m} \alpha_{i} x^{i}
$$

Here, $x$ and $X$ are the canonical coordinate and momentum, and the $\alpha_{i}$ are independent of $x$ and $X ; \alpha_{l}$ and $\alpha_{m}$ are assumed to be non-zero. The perturbation will be polynomial in $x$ and $X$,

$$
\mathcal{H}(x, X ; \varepsilon)=\sum_{n=0} \varepsilon^{n} \mathcal{H}_{n}(x, X),
$$

where $\varepsilon$ is a scaling parameter.

Using only polynomial arithmetic, we will transform the Hamiltonian into

$$
\mathcal{H}^{\prime}\left(x^{\prime}, X^{\prime} ; \varepsilon\right)=\frac{1}{2} X^{\prime 2}+\sum_{i=0}^{m} \alpha_{i}^{\prime}(\varepsilon) x^{\prime i},
$$

with $\alpha_{i}^{\prime}(\varepsilon)=\alpha_{i}+\mathcal{O}(\varepsilon)$. Ideally, one would like to reduce the system completely to

$$
\mathcal{H}^{\prime}=\mathcal{H}_{0}\left(x^{\prime}, X^{\prime} ; \alpha^{\prime}(\varepsilon)\right)
$$

that is, $i$ ranging from $l$ rather than 0 , thereby seemingly eliminating entirely the perturbation. In fact, as we shall show, we achieve this reduction in two cases: 1) when the potential $\mathcal{V}$ begins with a quadratic term $(l=2)$, and 2) when no monomials in the perturbation have degree lower than $l$.

Although this reduction requires the liberties of non-canonical transformations, the Hamiltonian character can be recovered (Henrard and Wauthier, 1988) by supplementing the transformation with a change of independent variable $t-\tau$. Letting the vector $\mathbf{x}^{\prime}$ include both coordinates and momenta, we first note that, in general, the differential equations are transformed as

$$
\frac{d \mathbf{x}^{\prime}}{d t}=\left\{\mathbf{x}^{\prime} ; \mathcal{H}^{\prime}\right\}=\left\{\mathbf{x}^{\prime} ; \mathbf{x}^{\prime}\right\} \frac{\partial \mathcal{H}^{\prime}}{\partial \mathbf{x}^{\prime}} .
$$

As the matrix of Poisson brackets is the negative of the inverse of the matrix of Lagrange brackets, we can also write

$$
\frac{\partial \mathcal{H}^{\prime}}{\partial \mathbf{x}^{\prime}}=-\left[\mathbf{x}^{\prime} ; \mathbf{x}^{\prime}\right] \frac{d \mathbf{x}^{\prime}}{d t} \text {. }
$$


But for one degree of freedom, the latter matrix is simply $\mathbf{J} \mathcal{K}$, where $\mathbf{J}$ is the usual skew symmetric matrix, and $\mathcal{K}$ is the scalar function

$$
\mathcal{K}=\frac{\partial x}{\partial x^{\prime}} \frac{\partial X}{\partial X^{\prime}}-\frac{\partial X}{\partial x^{\prime}} \frac{\partial x}{\partial X^{\prime}} .
$$

For a canonical transformation $\mathcal{K}=1$, but even when it is not, the transformation of independent variable given by $\mathrm{d} t=\mathcal{K} \mathrm{d} \tau$ recovers the Hamiltonian form as

$$
\frac{d \mathbf{x}^{\prime}}{d \tau}=\mathbf{J} \frac{\partial \mathcal{H}}{\partial \mathbf{x}^{\prime}}
$$

This recovery of Hamiltonian equations can be made for any non-canonical transformation of a one degree of freedom system. Moreover, for any near identity transformation, such as a Lie transformation, the function $\mathcal{K}$ will have 1 as its zeroth order term. Within the confines of perturbation theory, the time transformation, at least, will not be singular.

In Section 2, we describe the algorithm for mapping such perturbed Hamiltonians onto the class of the unperturbed parts to arbitrary order. The process by which the independent variable is transformed, and how explicit analytic solutions can be obtained, is outlined in Section 3. In Section 4, an extended example is presented. We analyze a perturbed Duffing oscillator, being one of the simplest involving elliptic functions.

The hyperbolic trajectories as well as both classes of periodic orbits are treated.

\section{Simplification}

The first stage of simplification by synchronization consists of eliminating from the perturbation all monomials not of the same form as those in the potential. This we do by a Lie transformation (Deprit, 1969; Henrard, 1970; Meyer, 1991) $(x, X) \leftarrow\left(x^{\prime}, X^{\prime}\right)$ using, as generator, the vector

$$
\mathbf{W}=\frac{d \mathbf{x}}{d \varepsilon}=\sum_{i \geq 0} \varepsilon^{i} \mathbf{W}_{i+1},
$$

where $\mathrm{x}$ has components $x$ and $X$ and $\mathbf{W}$ has components $W^{x}$ and $W^{X}$. The Lie derivative $\mathcal{L}_{W}$ of a scalar function $f(x, X)$ is

$$
\mathcal{L}_{W} f=\nabla f \cdot \mathbf{W}=\frac{\partial f}{\partial x} W^{x}+\frac{\partial f}{\partial X} W^{X} .
$$

At each order in the process of Lie simplification, we choose $\mathbf{W}_{n}$ to compute the simplified $\mathcal{H}_{n}^{\prime}$ via the differential identity (Deprit, 1969) 


$$
\mathcal{H}_{n}^{\prime}=\tilde{\mathcal{H}}_{n}+\mathcal{L}_{W_{n}} \mathcal{H}_{0}=\tilde{\mathcal{H}}_{n}+\frac{\partial \mathcal{V}}{\partial x} W_{n}^{x}+X W_{n}^{X} .
$$

Partitioning the tentative term $\tilde{\mathcal{H}}_{n}$ as the sum $X a(x, X)+b(x)$, then choosing $W_{n}^{X}=-a$ removes $X$ entirely from the perturbation. Writing

$$
b(x)=\sum_{i \geq 0} b_{i} x^{i}, \quad W_{n}^{x}=\sum_{i \geq 0} w_{i} x^{i},
$$

we then partition (9) as the sum

$$
\mathcal{H}_{n}^{\prime}=h_{0, l-2}+h_{l-1}+h_{l, m-1}+h_{m}+h_{m+1, \ldots}
$$

where

$$
\begin{aligned}
& h_{0, l-2}=\sum_{i=0}^{l-2} b_{i} x^{i} \\
& h_{l-1}=\left(b_{l-1}+l \alpha_{l} w_{0}\right) x^{l-1} \\
& h_{l, m-1}=\sum_{i=l}^{m-1}\left(b_{i}+\sum_{j=l}^{i+1} j \alpha_{j} w_{i-j+1}\right) x^{i} \\
& h_{m}=\left(b_{m}+\sum_{j=l}^{m} j \alpha_{j} w_{m-j-1}\right) x^{m} \\
& h_{m+1, \ldots}=\sum_{i>m}\left(b_{i}+\sum_{j=l}^{m} j \alpha_{j} w_{i-j+1}\right) x^{i} .
\end{aligned}
$$

The term $h_{0, l-2}$ is unaffected by the transformation. The terms $h_{l, m-1}$ and $h_{m}$ have the same form as the potential $\mathcal{V}$ and will be retained. Taking $w_{0}=-b_{l-1} /\left(l \alpha_{l}\right)$ sets $h_{l-1}=0$. The remaining term, $h_{m+1, \ldots}$ is eliminated as follows. Letting $k=\operatorname{deg} b$, if $k \leq m$, we simply take $w_{i}=0$ for $i>1$. Otherwise, we take $w_{i}=0$ for $i>k-m+1$ and compute $w_{k-m+1} \ldots w_{2}$ by downward recursion from $i=k$ to $i=m+1$ using

$$
w_{i-m+1}=-\frac{1}{m \alpha_{m}}\left(b_{i}+\sum_{j=l}^{m-1} j \alpha_{j} w_{i-j+1}\right) .
$$

This leaves us with

$$
\mathcal{H}_{n}^{\prime}=\sum_{i=0}^{l-2} b_{i} x^{i}+\sum_{i=l}^{m}\left(b_{i}+\sum_{j=l}^{\min (m, i+1)} j \alpha_{j} w_{i-j+1}\right) x^{i} .
$$


We should add that we still have free choice for $w_{1}$, which is involved in each coefficient in the second summation of (17). It could be left equal to zero, as we will do, or chosen to achieve some relationship among the coefficients.

Having carried out the simplification through any finite order, the sum of the main part and simplified perturbations (17) takes the form in the new variables

$$
\mathcal{H}^{\prime}\left(x^{\prime}, X^{\prime}\right)=\frac{1}{2} X^{\prime 2}+\sum_{i=0}^{l-2} \beta_{i} x^{\prime i}+\mathcal{V}\left(x^{\prime} ; \alpha^{\prime}\right),
$$

where the $\beta_{i}=\mathcal{O}(\varepsilon)$ (or higher) and $\alpha_{i}^{\prime}=\alpha_{i}+\mathcal{O}(\varepsilon)$.

Were it not for the additional terms in $\beta$, we would have effected a reduction of the system (3) to the form of the unperturbed part (2), in effect entirely eliminating the perturbation. Assuredly, our procedure makes no provision for eliminating these terms, yet for perhaps the most interesting dynamical systems these terms will not arise. The simplest case is when $l \leq 2$, eg. when the potential $\mathcal{V}$ starts with a quadratic term. The sum then contributes at worst a constant energy shift $\beta_{0}$. The other case is when the perturbation contains no terms whose degree is less than $l$ - a natural situation when the asymptotic scale is defined by expansions about the origin. Then, we can show that the simplified system will also have no terms whose degree in $x$ is less than $l$. Indeed, let mindeg $F$ denote the 'minimum degree' of a polynomial $F(x, X)$, that is, the integer $n$ such that $F(\lambda x, \lambda X)=$ $\lambda^{n}\left[f_{0}(x, X)+\lambda f_{1}(x, X)+\ldots\right]$ where $f_{0} \neq 0$. Clearly, if mindeg $\mathcal{H}_{1} \geq l$, the process yields mindeg $W_{1}^{X} \geq l-1$, mindeg $\mathcal{H}_{1}^{\prime} \geq l$ and mindeg $W_{1}^{x} \geq 1$ (since $b_{l-1}=0$ ). Further, the Lie derivative preserves this measure; if mindeg $F \geq$ $l$, then mindeg $\mathcal{L}_{W_{1}} F \geq l$. Consequently, mindeg $\tilde{\mathcal{H}}_{2} \geq l$, and, by induction, mindeg $\mathcal{H}_{i}^{\prime} \geq l$ for all $i$. In other words, for these two special classes, the most often encountered in fact, we have succeeded in transforming the perturbed system into the unperturbed one.

There is one caveat about the structure of the potential $\mathcal{V}$, although it should not be surprising. Even if $\alpha_{j}=0$ for some $j$ in $l<j<m$, the resulting $\alpha_{j}^{\prime}=\mathcal{O}(\varepsilon) \neq 0$, in general. For example, consider a main part which possesses reflection symmetry; the potential $\mathcal{V}$ contains only even powers of $x$; so $\alpha_{j}=0$ for odd $j$. If the perturbation is itself symmetric, containing only monomials of even degree, it can be shown that $\mathcal{H}^{\prime}$ will likewise be symmetric. On the other hand, when odd powers are present in the perturbation, we will, in general, have $\alpha_{j}^{\prime} \neq 0$ for some odd $j$. The procedure does not extend to the simplified system the reflection symmetry enjoyed by the unperturbed system.

\section{Analytic Solutions}

Regardless of the form achieved by the reduction, (18) or (5), we may, in principle, proceed to obtain explicit solutions as a function of time as follows. 
- Compute $\mathcal{K}\left(x^{\prime}, X^{\prime}\right)$ : With the generator $\mathbf{W}$ one calculates the Lie transformations $x\left(x^{\prime}, X^{\prime}\right), X\left(x^{\prime}, X^{\prime}\right)$, and by differentiation the elements in the determinant $\mathcal{K}\left(x^{\prime}, X^{\prime}\right)$ of (6). Note that $x, X, \mathcal{K}$ will be polynomial in $x^{\prime}, X^{\prime}$. Since $x=x^{\prime}+\mathcal{O}(\varepsilon)$ and $X=X^{\prime}+\mathcal{O}(\varepsilon)$, then $\mathcal{K}=1+\mathcal{O}(\varepsilon)$. [In (Henrard and Wauthier, 1988) a short-cut method of computing $\mathcal{K}$ during the simplification itself is described; this is handy when the explicit transformation is not needed.]

- Treat $\mathcal{H}^{\prime}$ as a Hamiltonian with the dependent variable being $\tau$. Solve the system for $x^{\prime}(\tau), X^{\prime}(\tau)$.

- Integrate $\mathcal{K}\left(x^{\prime}(\tau), X^{\prime}(\tau)\right)$ with respect to $\tau$ to obtain

$$
t=\tau+\Psi(\tau, \varepsilon)
$$

where $\Psi$ is first-order in $\varepsilon$.

- Invert the implicit equation $t=t(\tau)$ to obtain $\tau(t)$. This may be done efficiently using Lie transformations (Meyer, 1991), by noting that a transformation of a function of $\tau$ into a function of $t$ is achieved by a generator $W=d \tau / d \varepsilon$. The condition that $d t / d \varepsilon=0$ leads to the generator

$$
W=\frac{\partial \tau}{\partial \varepsilon}=-\frac{\partial \Psi / \partial \varepsilon}{1+\partial \Psi / \partial \tau}
$$

The full solutions then take the form

$$
x\left(x^{\prime}(\tau(t)), X^{\prime}(\tau(t))\right), \quad X\left(x^{\prime}(\tau(t)), X^{\prime}(\tau(t))\right) .
$$

It may be argued that carrying out all of these many steps may not be necessary for a given purpose, or even if it is needed, that it may not be possible. In particular, a Hamiltonian with a potential of high degree may not be solvable in closed form. Yet, we are able to keep more in the potential than is usually the case. Is it not more accurate to keep $x^{4}$ in the principal part and relegate $x^{6}$ to the perturbation, than to lose both?

In any case, each step in the outline above yields more information about the dynamics, and further steps are optional. For example, one may be content determining the shapes of the orbits; if so, one need not compute $\mathcal{K}$ and $\tau(t)$. Or one may be satisfied with just computing the trajectories and the real time as a function of the pseudo-time $r$; then $t(\tau)$ need not be inverted. If only the orbital periods are required, this computation can also be abbreviated.

Indeed, suppose $\mathbf{x}^{\prime}(\tau)$ is periodic in $\tau$ with period $\mathcal{T}$. Then $x, X$ and $\mathcal{K}$ are likewise periodic. The time transformation (19) decomposes into parts linear and periodic in $\tau$,

$$
t=\bar{\Psi} \tau+\tilde{\Psi}(\tau)
$$


The coefficient $\bar{\Psi}$ is the average of $\mathcal{K}$ over one period; it is of the form $1+\mathcal{O}(\varepsilon)$. The integral of the remainder $\mathcal{K}-\bar{\Psi}$ is $\tilde{\Psi}$; it is periodic with zero mean and is of first-order in $\varepsilon$. Hence $t(\tau)$ is pseudo-periodic in $\tau$. As $\tau$ advances by $\mathcal{T}, x$ and $X$ return to their original values, but $t$ advances by $\bar{\Psi} \mathcal{T}$. Thus, the period in the $t$ time-scale is $T=\bar{\Psi} \mathcal{T}$.

In the case of periodic solutions, we must be careful about using the generator (20). A naive transformation of $S=\sin (\omega \tau)$ using (20) will produce a secular expansion involving $\sin (\omega t)$. This result hides the true period of the expression, but it is not erroneous; it merely has a limited range of validity. To accommodate the distinction between periods in the two timescales, we recommend introducing an effective frequency $\omega^{\prime}=\omega / \bar{\Psi}$ and viewing (21) as an implicit equation

$$
t=\tau^{\prime}+\tilde{\Psi}^{\prime}\left(\tau^{\prime}\right), \quad \text { where } \tau^{\prime}=\bar{\Psi} \tau \text { and } \tilde{\Psi}^{\prime}\left(\tau^{\prime}\right)=\tilde{\Psi}\left(\tau^{\prime} / \bar{\Psi}\right),
$$

defining $\tau^{\prime}$ as a function of $t$. Then, any function of $\tau^{\prime}$ will be transformed into a function of $t$ by using

$$
W=\frac{\partial \tau^{\prime}}{\partial \varepsilon}=-\frac{\partial \tilde{\Psi}^{\prime} / \partial \varepsilon}{1+\partial \tilde{\Psi}^{\prime} / \partial \tau^{\prime}}
$$

as the generator. Thus, for instance, a function like $S=\sin (\omega \tau)=\sin \left(\omega^{\prime} \tau^{\prime}\right)$ will be transformed into a genuinely periodic expression involving $\sin \left(\omega^{\prime} t\right)$ with the correct period on the $t$ timescale.

\section{An extended example: The Duffing oscillator}

As an illustration of the simplification and analysis proposed here, we consider the Hamiltonian (1). The scaling

$$
(x, X, \beta, \mathcal{H}) \rightarrow(\sqrt[4]{\varepsilon} x, \sqrt[4]{\varepsilon} X, \beta / \sqrt{\varepsilon}, \sqrt{\varepsilon} \mathcal{H})
$$

puts (1) into the form

$$
\mathcal{H}=\frac{1}{2} X^{2}+\frac{\alpha}{2} x^{2}+\frac{\beta}{4} x^{4}+\varepsilon \frac{\gamma}{6} x^{6} .
$$

Here $\varepsilon$ is just a token to label the order; eventually it will be set to 1 . With the scaling which leads to a perturbed harmonic oscillator, the requirement that the perturbation be smaller than the main part, restricts the treatment to $x^{2} \ll|\alpha / \beta|$. In contrast, the above scaling extends the range to $x^{2} \ll|\beta / \gamma|$ and this will allow coverage of the equilibria near $\pm \sqrt{-\alpha / \beta}$.

The computations have been carried out in Macsyma (Symbolics, 1988) using a package for symbolic manipulation of Jacobian elliptic functions (publication of this package and its documentation is in preparation). 


\subsection{LIE SIMPLIFICATION}

Our simplification procedure, as described in Section 2, produces

$$
\mathcal{H}^{\prime}=\frac{1}{2} X^{\prime 2}+\frac{\alpha}{2} x^{\prime 2}+\frac{\beta^{\prime}}{4} x^{\prime 4}
$$

with

$$
\beta^{\prime}=\beta-\varepsilon \frac{2 \alpha \gamma}{3 \beta}-\varepsilon^{2} \frac{5 \alpha^{2} \gamma^{2}}{9 \beta^{3}}+\mathcal{O}\left(\varepsilon^{3}\right) .
$$

The expansion (25) will only be valid when $\beta^{2} \gg|\alpha \gamma|$, suggesting that in fact this method supplements rather than replaces the harmonic oscillator treatment, the latter requiring that $\beta$ be small. We point out that any perturbation containing only even powered monomials in $x$ and $X$ would yield exactly the same simplified Hamiltonian (24). Even though the expressions presented in the remainder of this section would be longer, the computations themselves would be essentially the same.

The generating function which obtained this simplification has components

$$
\begin{aligned}
& W^{x}=-\frac{\gamma}{6 \beta} x^{3}-\varepsilon \frac{\gamma^{2}}{18 \beta^{3}}\left(5 \alpha x^{3}-3 \beta x^{5}\right)+\mathcal{O}\left(\varepsilon^{2}\right), \\
& W^{X}=0+\mathcal{O}\left(\varepsilon^{2}\right),
\end{aligned}
$$

and the transformation is given by the equations

$$
\begin{aligned}
& x=x^{\prime}-\varepsilon \frac{\gamma}{6 \beta} x^{\prime 3}-\varepsilon^{2} \frac{\gamma^{2}}{72 \beta^{3}}\left(10 \alpha x^{\prime 3}-9 \beta x^{\prime 5}\right)+\mathcal{O}\left(\varepsilon^{3}\right), \\
& X=X^{\prime}+\mathcal{O}\left(\varepsilon^{3}\right) .
\end{aligned}
$$

The determinant establishing the time transformation is now easily computed:

$$
\mathcal{K}=1-\varepsilon \frac{\gamma}{2 \beta} x^{\prime 2}-\varepsilon^{2} \frac{5 \gamma^{2}}{24 \beta^{3}}\left(2 \alpha x^{\prime 2}-3 \beta x^{\prime 4}\right)+\mathcal{O}\left(\varepsilon^{3}\right) .
$$

\subsection{EXPLICIT SOLUTIONS FOR MAIN PART}

In considering the simplified Hamiltonian (24), we first notice that the system has an equilibrium $C_{0}$ at $x^{\prime}=X^{\prime}=0$ which is stable if $\alpha>0$. In addition, when $\alpha$ and $\beta^{\prime}$ have opposite signs, there is a pair of equilibria $C_{ \pm}$at $x_{ \pm}^{\prime}= \pm \sqrt{-\alpha / \beta^{\prime}}, X^{\prime}=0$; they are stable when $\alpha<0$. The energy at these points is $\mathcal{E}_{ \pm}=-\alpha^{2} /\left(4 \beta^{\prime}\right)$. We also notice that the system is symmetric with respect to the inversion $\left(x^{\prime}, X^{\prime}\right) \rightarrow$ $\left(-x^{\prime},-X^{\prime}\right)$.

As is well known, the solutions to (24) can be expressed in Jacobian elliptic 
functions (see, for example, (Bradbury, 1966)):

$$
x^{\prime}=r \operatorname{cn}(\omega \tau, k), \quad X^{\prime}=-r \omega \operatorname{sn}(\omega \tau, k) \operatorname{dn}(\omega \tau, k) .
$$

The constants $r, \omega$ and $k$ are determined by the initial conditions. At a given energy $\mathcal{E}$, the 'radius' $r$ is a root of the equation

$$
\mathcal{E}=\frac{1}{2} \alpha r^{2}+\frac{1}{4} \beta^{\prime} r^{4}
$$

one then computes the 'frequency' $\omega$ and the modulus $k$ from

$$
\omega^{2}=\alpha+\beta^{\prime} r^{2} \quad \text { and } \quad k^{2}=\frac{\beta^{\prime} r^{2}}{2 \omega^{2}} .
$$

That (32) is an even function of $r$ is to be expected from the inversion symmetry - both signs of $r$ are valid. But, except in the cases $\beta^{\prime}=0$ (where $r^{2}=2 \mathcal{E} / \alpha$ ) and $\mathcal{E}=0$ (where $r^{2}=-2 \alpha / \beta^{\prime}$; we exclude $r=0$ ), there are still two roots for $r^{2}$. In fact, the two roots give identical solutions, provided we make use of the arbitrary origin of $\tau$. If we take one root for $r^{2}$ along with its corresponding $\omega$ and $k$, that solution can be transformed into the other providing we offset the origin of $\tau$ by $\left(K(k)-i K^{\prime}(k)\right) / \omega$. The equivalence is proved using identities given in Byrd and Friedman (1954). [ $K(k)$ is the complete elliptic integral of the first kind, $K^{\prime}(k)=K\left(k^{\prime}\right)$, with $k^{\prime}=\sqrt{1-k^{2}}$, is the complement.] Consequently, we may take whichever solution to (32) is most convenient. We also note that $x^{\prime}$ and $X^{\prime}$ are even functions of $\omega$ and $k$, so the signs of $\omega$ and $k$ are irrelevant.

\subsection{Classification OF ORBITS}

For given initial conditions and parameters, the type of orbit of a Duffing system like (24) can be classified into one of four types or into separatrices between them. Witness Figure 1 (from Coppola (1989)), where, for different values of the parameters $\alpha$ and $\beta^{\prime}$, we display various kinds of phase flows.

One may think that the solutions of form (31) represent only the circulations about the origin. Quite the contrary, they are a most general form of the solution to the Duffing oscillator and cover virtually all situations encountered in Figure 1.

Circulations about the origin occur for positive energies when either $\beta^{\prime}>0$ or when $\beta^{\prime}=0$ and $\alpha>0$. These circulations also occur when $\alpha>0, \beta^{\prime}<0$ and $0<\mathcal{E}<\mathcal{E}_{ \pm}$, providing $x^{\prime}$ is initially in the range $\left[x_{-}^{\prime}, x_{+}^{\prime}\right]$. In these cases, one of the roots of (32) gives $r$ and $\omega$ real and $k^{2}<1$.

When $\alpha<0, \beta^{\prime}>0$, a separatrix surrounding the equilibria $C_{ \pm}$is approached in the limit $\mathcal{E}=0$. Below that energy, $0>\mathcal{E}>\mathcal{E}_{ \pm}$, there are two families of orbits circulating about the equilibria $C_{ \pm}$. In this range, the same root to (32) makes $r$ and $\omega$ real, but $k>1$.

The remainder of the parameter and energy space gives rise to orbits resembling 


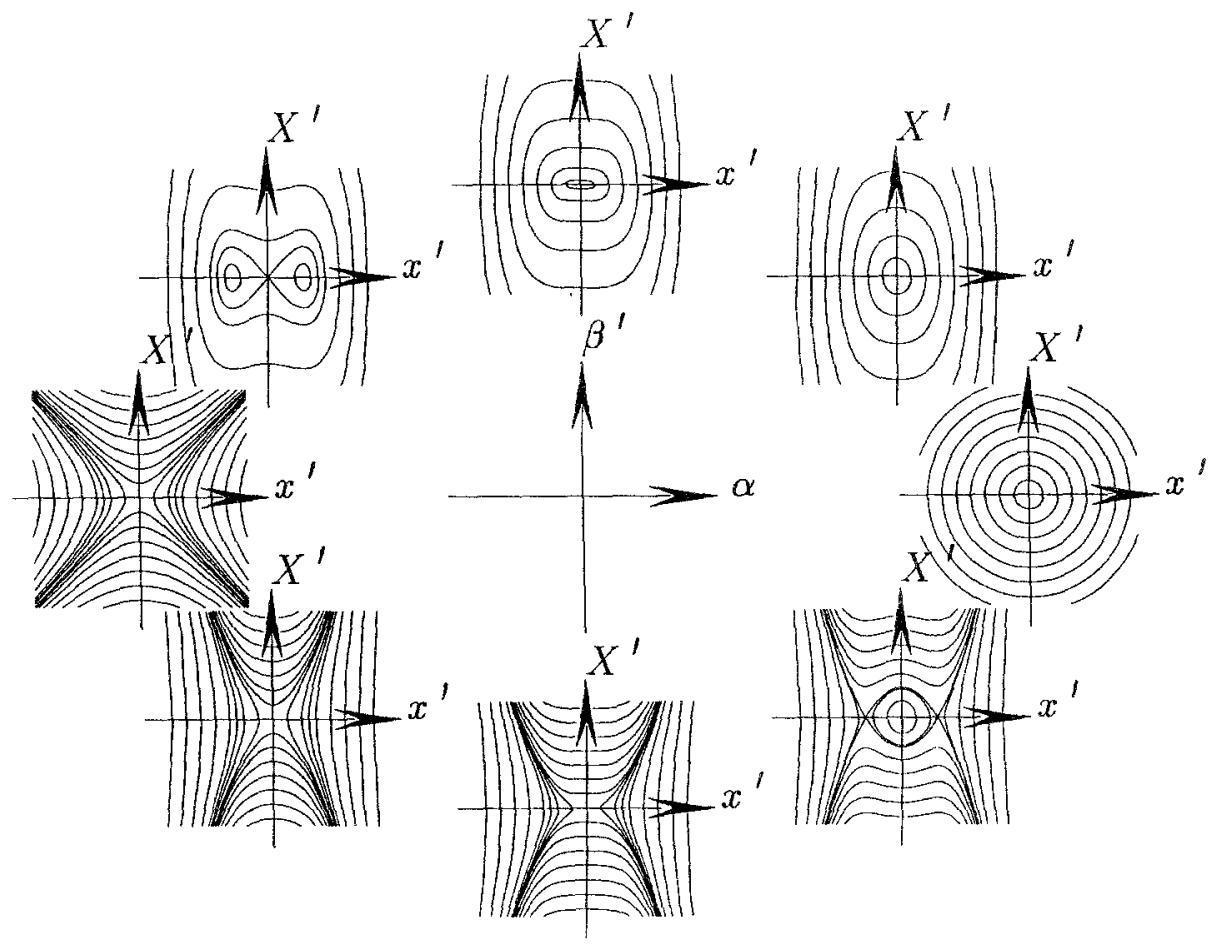

Fig. 1. Trajectories of the system (24) for various values of $\alpha$ and $\beta^{\prime}$.

hyperbolas which cross either the $x^{\prime}$ - or $X^{\prime}$-axis. In these cases, either root of (32) gives one or both of $r$ and $\omega$ complex, although the solutions (31) are still real valued. In particular, when the energy is negative and either i) $\beta^{\prime}<0$ or ii) $\beta^{\prime}=0$ and $\alpha<0$, the orbits cross the $x^{\prime}$-axis. In addition, when $\alpha>0, \beta^{\prime}<0$ and $0<\mathcal{E}<\mathcal{E}_{ \pm}$, if $x^{\prime}$ is initially outside the equilibria $C_{ \pm}$, the orbits are also of this class. Finally, in the remaining regions, that is for $\alpha<0, \beta^{\prime} \leq 0$ and $\mathcal{E}>0$ or $\alpha>0, \beta^{\prime}<0$ and $\mathcal{E}>\mathcal{E}_{ \pm}$, the trajectories are hyperbolas crossing the $X^{\prime}$-axis. Between these two classes of trajectories there are again separatrices. Only in the cases when $\mathcal{E}=0$ and either $\alpha=0$ or $\beta^{\prime}=0$, does the generic expression of the solution (31) fail to represent the separatrices.

In the following subsections we will show how our simplification procedure applies to each of these classes of orbit, including the separatrix between the two classes of circulations. We will also explain why we omit the separatrix between the hyperbolic trajectories. In light of (25), we will not discuss the case $\beta=0$ further.

In the interest of brevity, we will use the following shorthand notation 


$$
\begin{array}{lll}
s=\operatorname{sn}(u, \kappa), & d=\operatorname{dn}(u, \kappa), & K=K(\kappa), \\
c=\operatorname{cn}(u, \kappa), & Z=Z(u, \kappa), & E=E(\kappa) .
\end{array}
$$

The dependence of the quantities $u$ and $\kappa$ on $t, \tau, \omega$ and $k$ will vary and will be specified case by case. $[E(\kappa)$ is the complete elliptic integral of the second kind; $Z(u, \kappa)$ is the Jacobian zeta function.]

\subsection{Circulations about THE ORIGiN}

The most convenient root of (32) for the case of circulations about the origin is

$$
r^{2}=\frac{D-\alpha}{\beta^{\prime}}, \quad \omega^{2}=D \text { and } k^{2}=\frac{1}{2}\left(1-\frac{\alpha}{D}\right)
$$

where $D=\sqrt{\alpha^{2}+4 \beta^{\prime} \mathcal{E}}$. For this choice, $r$ and $\omega$ are real and $k^{2}$ is $<1$ (whether $k$ is real or imaginary causes no problems). Furthermore, $x^{l}$ and $X^{\prime}$ (and thus $x$ and $X$ ) have period $\mathcal{T}=4 K(k) / \omega$ on the time scale defined by $\tau$.

The connection $t(\tau)$ between time scales is obtained, as described in Section 3, by carrying out the integration and by partitioning the result. In the formula below we employ the shorthand (34) with $u=\omega \tau$ and $\kappa=k$.

$$
\begin{gathered}
\bar{\Psi}=1+\varepsilon \frac{\gamma r^{2}}{2 \beta k^{2}}\left[k^{\prime 2}-\frac{E}{K}\right]+\varepsilon^{2} \frac{5 \gamma^{2} r^{2}}{24 \beta^{3} k^{4}}\left[\beta k^{2} k^{\prime 2} r^{2}+\right. \\
\left.+2\left(\alpha k^{2}+\beta r^{2}\left(1-2 k^{2}\right)\right)\left(k^{\prime 2}-\frac{E}{K}\right)\right]+\mathcal{O}\left(\varepsilon^{3}\right), \\
\tilde{\Psi}(\tau)=-\varepsilon \frac{\gamma r^{2}}{2 \beta k^{2} \omega} Z+\varepsilon^{2} \frac{5 \gamma^{2} r^{2}}{24 \beta^{3} k^{4} \omega}\left[\beta k^{2} r^{2} s c d+\right. \\
\left.-2\left(\alpha k^{2}+\beta r^{2}\left(1-2 k^{2}\right)\right) Z\right]+\mathcal{O}\left(\varepsilon^{3}\right) .
\end{gathered}
$$

Expanding the expression for the effective frequency, $\omega^{\prime}=\omega / \bar{\Psi}$, we obtain

$$
\begin{aligned}
\omega^{\prime}=\omega-\varepsilon \frac{\gamma r^{2} \omega}{2 \beta k^{2}}[ & \left.k^{\prime 2}-\frac{E}{K}\right]-\varepsilon^{2} \frac{\gamma^{2} r^{2} \omega}{24 \beta^{3} k^{4}}\left[k^{\prime 2}\left(10 \alpha k^{2}+\beta r^{2}\left(4-9 k^{2}\right)\right)+\right. \\
& \left.-2\left(5 \alpha k^{2}-\beta r^{2}\left(1+4 k^{2}\right)\right) \frac{E}{K}-6 \beta r^{2} \frac{E^{2}}{K^{2}}\right]+\mathcal{O}\left(\varepsilon^{3}\right)
\end{aligned}
$$

where we use the shorthand (34) with $u=\omega^{\prime} t$ and $\kappa=k$. The period in the $t$ time scale is thus $T=4 K(k) / \omega^{\prime}$. The expressions for $x^{\prime}$ and $X^{\prime}$ are reexpressed as functions of $t$ using the strategy described in Section 3 for periodic functions using the generator (22). 


$$
\begin{aligned}
& x^{\prime}=r c-\varepsilon \frac{\gamma r^{3}}{2 \beta k^{2}} Z s d+\varepsilon^{2} \frac{\gamma^{2} r^{3}}{24 \beta^{3} k^{4}}\left[5 \beta r^{2} k^{2} c s^{2} d^{2}+\right. \\
&-2\left(5\left(\alpha k^{2}+\beta r^{2}\left(1-2 k^{2}\right)\right)+3 \beta r^{2} k^{2} c^{2}\right) Z s d+ \\
&\left.+3 \beta r^{2}\left(1-2 d^{2}\right) Z^{2} c\right]+\mathcal{O}\left(\varepsilon^{3}\right), \\
& X^{\prime}=-r \omega s d+\varepsilon \frac{\gamma r^{3} \omega}{2 \beta k^{2}}\left(1-2 d^{2}\right) Z c+\varepsilon^{2} \frac{\gamma^{2} r^{3} \omega}{24 \beta^{3} k^{4}}\left[5 \beta r^{2}\left(2 d^{2}-1\right) c^{2} s d+\right. \\
&+2\left(5\left(\alpha k^{2}+\beta r^{2}\left(1-2 k^{2}\right)\right)+3 \beta r^{2} k^{2} c^{2}\right)\left(1-2 d^{2}\right) Z c+ \\
&\left.+3 \beta r^{2}\left(1+4 k^{2}-6 k^{2} s^{2}\right) Z^{2} s d\right]+\mathcal{O}\left(\varepsilon^{3}\right) .
\end{aligned}
$$

When $\alpha<0$, there exists a separatrix at $\mathcal{E}=0$ which encloses the circulations about the two equilibria $C_{ \pm}$. In the limit $\mathcal{E} \rightarrow 0$, from (35), $r^{2} \rightarrow-2 \alpha / \beta^{\prime}, \omega^{2} \rightarrow|\alpha|$ and $k \rightarrow 1$; also $\omega^{\prime} \rightarrow \omega$. Using the notation $s=\sinh (\omega t), c=\cosh (\omega t)$, the solutions become

$$
\begin{gathered}
x^{\prime}=\frac{r}{c}-\varepsilon \frac{\gamma r^{3}}{2 \beta} \frac{s^{2}}{c^{2}}-\varepsilon^{2} \frac{\gamma^{2} r^{3}}{24 \beta^{3}} \frac{s^{2}}{c^{5}}\left[7 \beta r^{2}+\left(10 \alpha-13 \beta r^{2}\right) c^{2}\right]+\mathcal{O}\left(\varepsilon^{3}\right), \\
X^{\prime}=-r \omega \frac{s}{c^{2}}-\varepsilon \frac{\gamma \omega r^{3}}{2 \beta} \frac{\left(2-c^{2}\right) s}{c^{4}}-\varepsilon^{2} \frac{\gamma^{2} r^{3} \omega}{24 \beta^{3}} \frac{s}{c^{6}}\left[10 \alpha-9 \beta r^{2}+\right. \\
\left.-16 \beta r^{2} s^{2}-\left(10 \alpha-13 \beta r^{2}\right) s^{4}\right]+\mathcal{O}\left(\varepsilon^{3}\right) .
\end{gathered}
$$

\subsection{CiRCULations InSIDE Figure-OF-8}

Within the separatrix discussed above, the modulus $k>1$ and the circulations are about the two equilibria $C_{ \pm}$; they are distinguished by taking different signs for $r$. Admittedly, the quantity $4 K(k) / \omega$ is a period of the solutions, but it is complex when $k>1$, and so the treatment must be amended to obtain the period corresponding to real time.

By applying the reciprocal modulus transformation we deduce from (31) that

$$
x^{\prime}=r \operatorname{dn}\left(\omega_{2} \tau, k_{2}\right) \quad X^{\prime}=-k_{2}^{2} r \omega_{2} \operatorname{sn}\left(\omega_{2} \tau, k_{2}\right) \operatorname{cn}\left(\omega_{2} \tau, k_{2}\right),
$$

where $\omega_{2}=k \omega, k_{2}=1 / k$ and $0<k_{2}<1$ (Byrd and Friedman, 1954, formula 162.01). Since the period is $\mathcal{T}=2 K\left(k_{2}\right) / \omega_{2}, t(\tau)$ is partitioned differently into linear and periodic parts. For brevity, we give the results only through first order.

$$
\bar{\Psi}=1-\varepsilon \frac{\gamma r^{2}}{2 \beta} \frac{E\left(k_{2}\right)}{K\left(k_{2}\right)}+\mathcal{O}\left(\varepsilon^{2}\right), \quad \tilde{\Psi}=-\varepsilon \frac{\gamma r^{2}}{2 \beta \omega_{2}} Z\left(\omega_{2} \tau, k_{2}\right)+\mathcal{O}\left(\varepsilon^{2}\right) .
$$


Proceeding as before, we obtain the effective frequency $\omega_{2}^{\prime}=\omega_{2} / \bar{\Psi}$ and solutions as a function of $t$. Here we use the shorthand (34) with $u=\omega_{2}^{t} t$ and $\kappa=k_{2}$.

$$
\begin{aligned}
& \omega_{2}^{\prime}=\omega_{2}+\varepsilon \frac{\gamma r^{2} \omega_{2}}{2 \beta} \frac{E}{K}+\mathcal{O}\left(\varepsilon^{2}\right), \\
& x^{\prime}=r d-\varepsilon \frac{\gamma r^{3} k_{2}^{2}}{2 \beta} s c Z+\mathcal{O}\left(\varepsilon^{2}\right), \\
& X^{\prime}=-k_{2}^{2} r \omega_{2} s c-\varepsilon \frac{\gamma r^{3} \omega_{2} k_{2}^{2}}{2 \beta} d Z\left(1-2 s^{2}\right)+\mathcal{O}\left(\varepsilon^{2}\right) .
\end{aligned}
$$

The period on the $t$ time-scale is $2 K\left(k_{2}\right) / \omega_{2}^{\prime}$.

In the limit $\mathcal{E} \rightarrow 0, k_{2}=1, \omega_{2}^{\prime}=\omega$. The orbits inside the figure-of- 8 approach the separatrix discussed in the previous subsection; in fact, in the limit, the above solutions become identical to those obtained in the limit in Subsection 4.4.

\subsection{HYPERBOLIC TRAJECTORIES CROSSING THE $x^{\prime}$-AXIS}

The simplified Hamiltonian (24) covers not only the periodic motions but also the hyperbolic motions that arise with $\beta^{\prime}<0$. The most convenient root of (32) for those orbits crossing $x^{\prime}$-axis is that for which

$$
r^{2}=-\frac{D+\alpha}{\beta^{\prime}}, \quad \omega^{2}=-D \text { and } k^{2}=\frac{1}{2}\left(1+\frac{\alpha}{D}\right),
$$

with $D=\sqrt{\alpha^{2}+4 \beta^{\prime} \mathcal{E}}$. Under these conditions, $r$ is real, $\omega$ is imaginary and $k>0$. In addition, $k \leq 1$ for $\mathcal{E} \leq 0$ but $k$ becomes greater than 1 when $\mathcal{E}$ is positive. The imaginary argument transformation (Byrd and Friedman, 1954, formula 161.01) re-expresses the solutions in terms of $\omega_{3}=-i \omega$ and $k_{3}=k^{\prime}$ such that $\omega_{3}$ is real, and $k_{3}^{2}<1$ at every energy level.

$$
x^{\prime}=\frac{r}{\operatorname{cn}\left(\omega_{3} \tau, k_{3}\right)}, \quad X^{\prime}=\frac{r \omega_{3} \operatorname{sn}\left(\omega_{3} \tau, k_{3}\right) \operatorname{dn}\left(\omega_{3} \tau, k_{3}\right)}{\operatorname{cn}^{2}\left(\omega_{3} \tau, k_{3}\right)} .
$$

By integration we obtain, using the shorthand with $u=\omega_{3} \tau$ and $\kappa=k_{3}$,

$$
t=\tau-\varepsilon \frac{\gamma r^{2}}{2 \beta \omega_{3} k_{3}^{\prime 2}}\left[\left(k_{3}^{\prime 2}-\frac{E}{K}\right) \omega_{3} \tau-Z+\frac{s d}{c}\right]+\mathcal{O}\left(\varepsilon^{2}\right) .
$$

Since the orbits escape to infinity, one should not be concerned with possible secular terms. The expansions will not be valid for very long times anyway. We can be content with inverting $t(\tau)$ on the basis of (20) to get $\tau(t)$. We express the solutions using the shorthand with $u=\omega_{3} t$ and $\kappa=k_{3}$ 


$$
\begin{aligned}
& x^{\prime}=\frac{r}{c}+\varepsilon \frac{\gamma r^{3}}{2 \beta k_{3}^{\prime 2}} \frac{s d}{c^{2}}\left[\left(k_{3}^{\prime 2}-\frac{E}{K}\right) \omega_{3} t-Z+\frac{s d}{c}\right]+\mathcal{O}\left(\varepsilon^{2}\right), \\
& X^{\prime}=\frac{r \omega_{3} s d}{c^{2}}+\varepsilon \frac{\gamma r^{3} \omega_{3}}{2 \beta k_{3}^{\prime 2}} \frac{2 d^{2}-c^{2}}{c^{3}}\left[\left(k_{3}^{\prime 2}-\frac{E}{K}\right) \omega_{3} t-Z+\frac{s d}{c}\right]+\mathcal{O}\left(\varepsilon^{2}\right) .
\end{aligned}
$$

In order to obtain equations for the separatrices $\left(\mathcal{E}=0\right.$ for $\alpha<0$ and $\mathcal{E}=\mathcal{E}_{ \pm}$ for $\alpha>0$ ), one may be tempted to take the limit as the energy approaches either of these values. But, the orbits simply reduce to the appropriate unstable equilibrium ( $C_{0}$ or $C_{ \pm}$, respectively). To obtain curves as a non-trivial function of time one must first shift the origin of $t$ so that $t=0$ corresponds not to the equilibrium but to the point at infinity. Even doing that, however, with the perturbation procedure we have presented, there is little hope of obtaining useful results for these separatrices. Since we are limited to a neighborhood of the origin, we will only cover that part of the separatrix which is at or near the fixed points. The motion near the unstable fixed points is extremely slow, however, and so the secular expansions will, in effect, become invalid before the object has made any observable motion.

\subsection{HYPERBOLIC TRAJECTORIES CROSSING THE $X^{\prime}$-AXIS}

The remaining regions of parameter and phase space are filled with hyperbolic trajectories that cross the $X^{\prime}$-axis. We find that either root for the three quantities $r, \omega$ and $k$ are complex, which complicates significantly the treatment. In order to put the solutions (31) into a more convenient form, we will proceed through a sequence of transformations. One problem is that (31) expresses $x^{\prime}$ in terms of $\mathrm{cn}$, an even function of $\tau$, whereas for the orbits under consideration, $x^{\prime}$ is an odd function of $\tau$. By shifting the origin of $\tau$ by the quarter period $K(k) / \omega$ we obtain

$$
x^{\prime}=-\frac{r k^{\prime} \operatorname{sn}(\omega \tau, k)}{\operatorname{dn}(\omega \tau, k)}, \quad X^{\prime}=-\frac{r \omega k^{\prime} \operatorname{cn}(\omega \tau, k)}{\mathrm{dn}^{2}(\omega \tau, k)},
$$

alleviating this concern. To obtain demonstrably real expressions, we continue by applying a transformation given in formula 165.05 in (Byrd and Friedman, 1954),

$$
x^{\prime}=-\frac{r_{4} \operatorname{sn}\left(\omega_{4} \tau, k_{4}\right)}{\operatorname{cn}\left(\omega_{4} \tau, k_{4}\right) \operatorname{dn}\left(\omega_{4} \tau, k_{4}\right)}, \quad X^{\prime}=-\frac{r_{4} \omega_{4}\left(1-k_{4}^{2} \operatorname{sn}^{4}\left(\omega_{4} \tau, k_{4}\right)\right)}{\mathrm{cn}^{2}\left(\omega_{4} \tau, k_{4}\right) \mathrm{dn}^{2}\left(\omega_{4} \tau, k_{4}\right)},
$$

where $k=\left(1+k_{4}\right) /\left(2 \sqrt{k_{4}}\right), \omega=2 \sqrt{k_{4}} \omega_{4}$ and $r_{4}=i r\left(1-k_{4}\right)$. The useful roots for these conditions, using (38), are

$$
r_{4}^{2}=\frac{8 E}{\sqrt{-4 \beta^{\prime} \mathcal{E}}-\alpha}, \quad \omega_{4}^{2}=\frac{1}{4}\left(\sqrt{-4 \beta^{\prime} \mathcal{E}}-\alpha\right), \quad k_{4}^{2}=\frac{\alpha+\sqrt{-4 \beta^{\prime} \mathcal{E}}}{\alpha-\sqrt{-4 \beta^{\prime} \mathcal{E}}},
$$

for these make $r_{4}, \omega_{4}$ and $k_{4}^{2}$ all real, and $k_{4}^{2}<1$, in the regions of interest. 
Integration yields the time transformation $\left(u=\omega_{4} \tau, \kappa=k_{4}\right)$,

$$
t=\tau-\varepsilon \frac{\gamma r_{4}^{2}}{2 \beta k_{4}^{\prime 4} \omega_{4}}\left[\left(k_{4}^{\prime 2}-2 \frac{E}{K}\right) \omega_{4} \tau-2 Z+2 \frac{\left(2 d^{2}-k^{\prime 2}\right) s}{c d}\right]+\mathcal{O}\left(\varepsilon^{2}\right) .
$$

The solutions are then re-expressed as functions of $t,\left(u=\omega_{4} t, \kappa=k_{4}\right)$

$$
\begin{aligned}
& x^{\prime}=-\frac{r_{4} s}{c d}- \varepsilon \frac{\gamma r_{4}^{3}}{2 \beta k_{4}^{\prime 4}} \frac{1-k_{4}^{2} s^{4}}{c^{2} d^{2}} \times \\
& \times\left[\left(k_{4}^{\prime 2}-2 \frac{E}{K}\right) \omega_{4} t-2 Z+\frac{\left(2 d^{2}-k^{\prime 2}\right) s}{c d}\right]+\mathcal{O}\left(\varepsilon^{2}\right), \\
& X^{\prime}=- \frac{r_{4} \omega_{4}\left(1-k_{4}^{2} s^{4}\right)}{c^{2} d^{2}}-\varepsilon \frac{\gamma r_{4}^{3} \omega_{4}}{\beta k_{4}^{\prime 4}} \frac{\left(k^{2} c^{4}+d^{4}\right) s}{c^{3} d^{3}} \times \\
& \times\left[\left(k_{4}^{\prime 2}-2 \frac{E}{K}\right) \omega_{4} t-2 Z+\frac{\left(2 d^{2}-k^{\prime 2}\right) s}{c d}\right]+\mathcal{O}\left(\varepsilon^{2}\right) .
\end{aligned}
$$

\subsection{COMPARISONS}

To validate our techniques we have compared orbits computed as we have prescribed to the results of numerical integration of the original differential equations. We have used a Bulirsch-Stoer integrator (see for example (Press et al., 1988)) in IEEE double-precision. Each step of the integrator produced a value for $t$ and the position $\mathbf{x}_{i}$. We then computed the position $\mathbf{x}_{c}$ for the time $t$ from the appropriate formula derived above and, thus, the relative error $\Delta \mathbf{x}=\left(\mathbf{x}_{i}-\mathbf{x}_{c}\right) /\left|\mathbf{x}_{i}\right|$.

Two kinds of errors of computed orbits are of particular interest: 1) the extent to which the orbit looses synchronization with the true orbit; and 2) the extent to which the computed orbit wanders away from its energy manifold. In order to measure these two errors independently we have computed the relative error both tangent to the trajectory, $\varepsilon_{T}=(\Delta \mathrm{x} \cdot \dot{\mathrm{x}}) /|\dot{\mathrm{x}}|$, and normal to it, $\varepsilon_{N}=(\Delta \mathrm{x} \times \dot{\mathrm{x}}) /|\dot{\mathrm{x}}|$. In general, we would expect $\varepsilon_{T}$ to show a degradation over long periods due to the truncated approximation to the frequencies of the system (Deprit and Rom, 1969), and indeed this error should eventually become quite large due to the asymptotic nature of the expansions. On the other hand, $\varepsilon_{N}$ should remain small and flat for a long period of time.

In Figure 2, we show the error for orbits which circulate about the origin, using the formula from Subsection 4.4. The logarithms of these errors are shown as a function of time for various orders of approximation. The features are as expected; the short period variations on each curve can be attributed to missing periodic contributions from the next omitted order. The approximations appear to retain their accuracy for relatively long times; the fourth-order approximation remains accurate to one part in $10^{4}$ for over ten periods. 

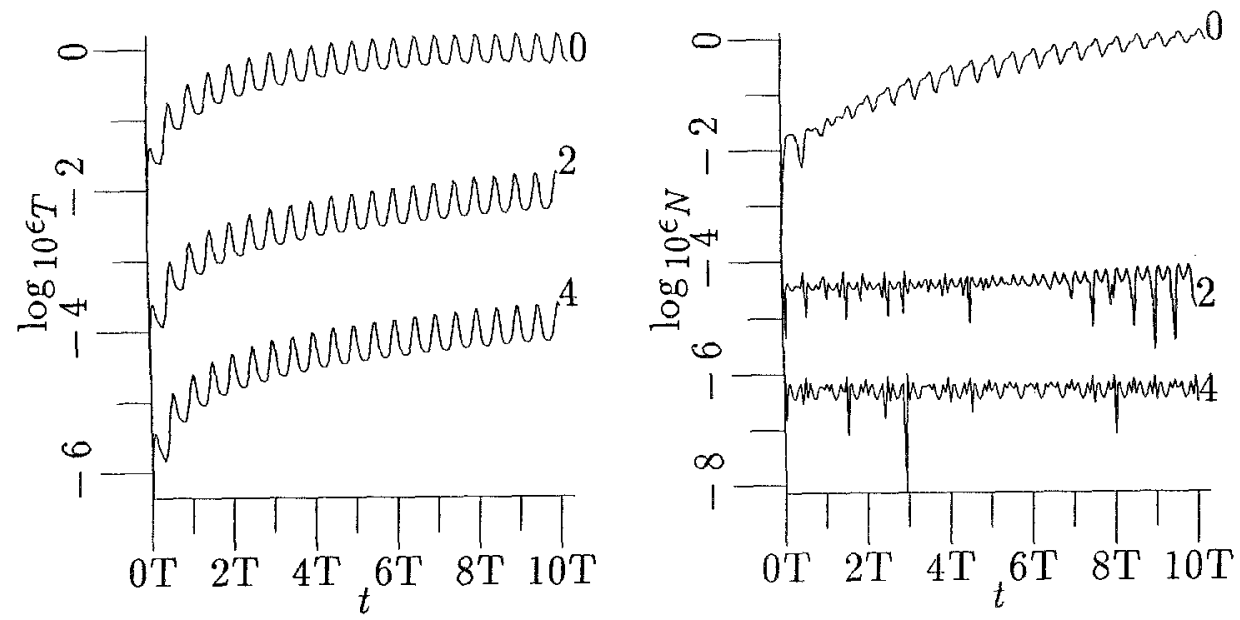

Fig. 2. Logarithm of error between computed and integrated orbits as a function of time over 10 periods. The orbits are circulations about the origin starting at $(1.5,0)$ for $\alpha=1, \beta=1$ and $\gamma=1 / 20$. The errors $\varepsilon_{T}$ and $\varepsilon_{N}$ are the relative errors tangent to and normal to the trajectory (see text). The number at the right of each curve indicates the order of approximation used.

To put Figure 2 into perspective, we compared the results of our procedure with the orbits that one computes from the usual normalization of (1) as a perturbed harmonic oscillator in action-angle variables. We show this comparison in Figure 3 where we have displayed only the magnitude of the relative error $\varepsilon=|\Delta \mathbf{x}|$; but since the initial condition used in Figure $2(x=1.5, X=0)$ is well outside the domain of validity for the harmonic oscillator, we have used ( $x=0.5, X=0$ ) for this figure. The disadvantage of the harmonic oscillator treatment is apparent; a fourth-order approximation must be used to represent the zeroth-order approximation in the synchronized approach - that is, simply to approximate the elliptic functions with $\gamma$ neglected. A ninth- or tenth-order approximation is equivalent to only a second-order approximation in the current method. Even with the complications of the elliptic functions, such low-order expansions are easily carried out within Macsyma, but to obtain the high-order approximations for the perturbed harmonic oscillator we found it advantageous to use our special purpose system MAO.

Finally, in Figure 4 we verify that the approximations obtained for non-periodic orbits, in this case the hyperbolic orbits crossing the $x^{\prime}$ axis, are also quite good. Here, we have plotted the components of error versus the distance from the origin; the fourth-order approximation remains accurate to one part in $10^{4}$ within a disk of radius 5 . However, it is clear that the formula do not preserve the energy constant as well as for the previous case. That the various approximations appear to cross around 10 demonstrates a point made by Sanders and Verhulst (1985), namely that increasing the order may well increase the accuracy of an asymptotic expansion, but it does not lengthen the interval of validity. 

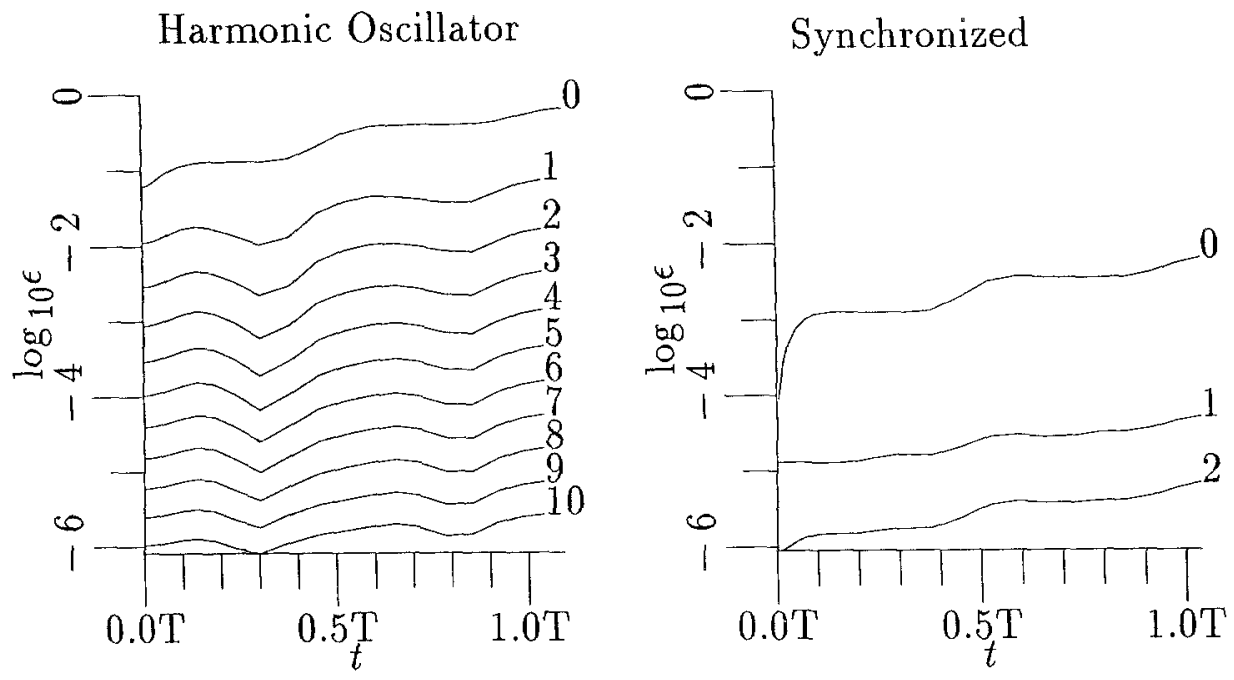

Fig. 3. Comparison between the errors of a perturbed harmonic oscillator (left) treatment to those of the proposed treatment (right) over 1 period. The error $\varepsilon$ is the total relative error. The orbits are circulations about the origin using the same parameters as 2 , but starting at $(0.5,0)$.
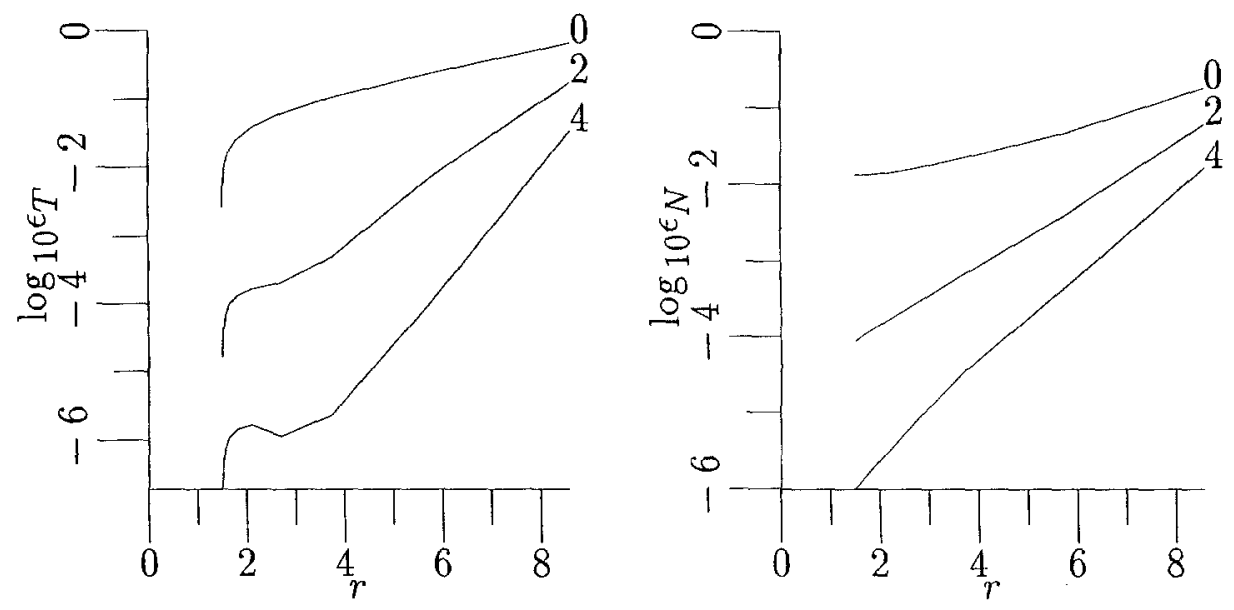

Fig. 4. Logarithm of error between computed and integrated orbits as a function of the distance from the origin. The orbits are hyperbolic trajectories crossing the $x^{\prime}$-axis starting at $(1.5,0)$ for $\alpha=-1$, $\beta=-1$ and $\gamma=1 / 20$. The errors $\varepsilon_{T}$ and $\varepsilon_{N}$ are the relative errors tangent to and normal to the trajectory (see text).

\section{Conclusions}

At a recent IAU symposium on the use of computers in dynamical astronomy, we expressed concern about a paradox of computing. Even as, or perhaps because, 
computers become faster, memories larger and software more efficient, one is driven to attack larger and more intricate problems. Yet the reach always exceeds the grasp. Even a computation as simple as normalizing a perturbed harmonic oscillator becomes expensive as the requirement on the order of approximation increases without apparent bound. We drew the attention of our colleagues to the need to explore different strategies for simplification.

We call a 'Lie simplification' any Lie transformation undertaken for the purpose of simplifying a perturbed dynamical system, in any sense that the problem might justify. By their very nature as near-identity transformations, Lie simplifications are tied to the structure of the principal part. A normalization in the classical sense is simply an extreme form of simplification: it eliminates from the perturbation all terms in the image of the Lie derivative associated with the principal part. This tends to remove the bulk of the terms from the perturbation, but at a cost that grows exponentially with the order of approximation. Perhaps worse, the inversion of the Lie derivative, not to mention the separation of image and kernel, can be quite unwieldy, especially when the solution of the principal part involves non-elementary functions. As a result, one often dodges the difficulty by moving troublemaking terms from the principal part into the perturbation. As we have shown, the artifice is not satisfactory. But are there alternatives?

Deprit's 'elimination of the parallax' points to one alternative. He replaced a single, difficult, normalization by a pair of easier Lie simplifications. The first eliminates only a subset of the image and is thus not a normalization. This aids in the subsequent normalization since the expressions are kept more manageable and thus the process can be carried to higher order.

Another alternative is the one we propose here, namely a Lie simplification to eliminate from the perturbation all terms of a type not present in the principal part. Since the perturbed system is replaced by a modified version of the principal part, it, in effect, completely eliminates the perturbation. We like to call such a simplification a synchronization. Indeed, it consists of a non-canonical transformation which reduces the perturbed system to the unperturbed, thereby connecting the motions of the two systems with a time transformation acting as the synchronizing clock. The concept was inspired by ideas found in recent papers by Deprit, Howland, Henrard and Wauthier, who were dealing with perturbed pendula. They sought to avoid, or at least postpone, explicit introduction of elliptic functions into the computations.

As a general strategy, simplification by synchronization has advantages over a classical normalization. The former proceeds in the algebra of the Hamiltonian, the latter in the algebra of the solutions of the main part. The former lacks the complications involving kernels, images and inversion of Lie derivatives. True, the construction of the synchronizing transformation does involve the algebra of the solutions, but in operations significantly less cumbersome than those required by a normalization. Besides, the construction is unnecessary if only an understanding of the phase flows is desired.

We found the concept of simplification by synchronization first realized for 
perturbed pendula. Here we offer a second application, broader in scope, to polynomial Hamiltonians. We anticipate this strategy being successfully applied to other difficult problems. The idea is worth exploring. There are so many dynamical systems, some quite interesting, that a strict normalization can crack only with great difficulty, if at all.

\section{Acknowledgements}

The authors would like to thank André Deprit for many worthwhile discussions on the subject. This work was supported in part by the Applied Mathematics Program at the Defense Advanced Research Program Agency (Washington, D.C.) (B.R.M.) and by the Office of Naval Technology's Postdoctoral Fellowship Program (Naval Research Laboratory, Washington, D.C.) (V.T.C.).

\section{References}

Bradbury, T.C.: 1966, Theoretical Mechanics, John Wiley \& Sons, New York.

Byrd, P.F. and Friedman, M.D.: 1954, Handbook of Elliptic Integrals for Engineers and Physicists, Springer-Verlag, Berlin.

Coppola, V.T.: 1989, Ph.D. thesis, Cornell University.

Deprit, A.: 1969, Celestial Mechanics 1, 12.

Deprit, A. and Rom, A.: 1969, Celestial Mechanics 2, 166.

Henrard, J.: 1970, Celestial Mechanics 3, 107.

Henrard, J. and Wauthier, P.: 1988, Celestial Mechanics 44, 227.

Howland, R.A.: 1988a, Celestial Mechanics 44, 209.

Howland, R.A.: 1988b, Celestial Mechanics 45, 407.

Meyer, K.R.: 1991, in: Computer Aided Proofs in Analysis, pp. 190-210, Springer-Verlag.

Press, W.H., Flannery, B.P., Teukolsky, S.A. and Vetterling, W.T.: 1988, Numerical Recipes in C, Cambridge University Press.

Sanders, J.A. and Verhulst, F.: 1985, Averaging Methods in Nonlinear Dynamical Systems, SpringerVerlag.

Symbolics: 1988, Macsyma Reference Manual, Symbolics Inc., Burlington, MA. 